was significantly associated with LBP, even when adjusted for other known predictive factors. Thus, preventive initiatives for low back pain among healthcare workers must focus on limiting manual patient handling and include the use of proper mechanical patient handling equipment and training on the use of these equipment.

\section{P.3.05 MARITIME FATAL ACCIDENTS AND VESSEL DISASTERS IN TAIWANESE FISHING VESSELS, 2003-2015}

1,2Ping-Hui Chen, 1,2Pau-Chung Chen. ${ }^{1}$ Department of Environmental and Occupational Medicine, National Taiwan University Hospital, Taipei, Taiwan; ${ }^{2}$ Institute of Occupational Medicine and Industrial Hygiene, National Taiwan University School of Public Health, Taipei, Taiwan

\subsection{6/OEM-2019-EPI.268}

Introduction Fishery is a hazardous industry with high occupational fatalities, mainly due to vessel disasters, especially among smaller vessels, according to European and NorthAmerican studies. However, Asian countries with different industry status and larger portion of global marine capture production are short of adequate investigation.

Methods In Taiwan, Fisheries Agency provided compensation for maritime fatalities and capsizing vessels, and recorded all enrolled crews and fishing vessels in Fishery Administration Management Information System. Using these two databases, incidence rate and odds ratio (OR) were calculated to depict an overall picture of maritime fatal accidents and associated causal factors.

Results From 2003 to 2015, there were 562 cases of fatal accidents, whose mechanisms were man overboard (368, $65.5 \%)$, followed by capsizing $(53,9.4 \%)$. Overall incidence rate was 3.6 per 10000 man-labour year. The rates were $2.51,4.12$, and 7.28 per 10000 man-labour year, and odds ratios were $1.0,1.64$ and 2.90 , for coastal $(<12$ Nautical miles, Nm), inshore (12-200 Nm), and deep sea (>200 Nm) fisheries.

There were 632 cases of vessel capsizing, whose mechanisms were fire $(162,25.63 \%)$, followed by natural disaster, mechanical problem $(85,13.45 \%)$, and collision $(71,11.23 \%)$. Overall incidence rate was 152.01 per 10000 vessels. The rates were $7.15,21.42,71.48$, and 51.95 per 10000 vessels, and odds ratios were 1.0, 3.00, 10.05 and 7.29, for smallsized (sampan and fishing raft), small-medium-sized $(<20$ gross registered tonnages, GRT), medium-large-sized (20-200 GRT) and large-sized (>200 GRT) vessels.

Conclusion Our findings showed the mixed effect of vessel size and fishery types on maritime fatal accidents, and deepsea medium-large-sized vessels, as the smallest vessels in deep sea fisheries, had the highest risk. Compared with other developed countries, more than half fishing vessels of deep sea fisheries in Taiwan are less than 100 GRT, and preventive intervention should be focused on these vessels.

\section{P.3.06 MARITIME FATALITIES DUE TO ACUTE ILLNESS IN TAIWANESE FISHING VESSELS, 2003-2015}

\footnotetext{
${ }^{1,2}$ Ping Hui Chen, ${ }^{1,2}$ Pau-Chung Chen. 'Department of Environmental and Occupational Medicine, National Taiwan University Hospital, Taipei, Taiwan; ${ }^{2}$ Institute of Occupational Medicine and Industrial Hygiene, National Taiwan University School of Public Health, Taipei, Taiwan
}

10.1136/OEM-2019-EPI.269
Introduction Fishery is notorious for its high maritime fatalities. Apart from fatal accidents, acute illness without timely emergency medical care may be another important reason for maritime fatalities. However, compared with fatal accidents, maritime fatalities due to acute illness are much less discussed and investigated, and there is need for epidemiological evidence to support and develop preventive strategies.

Methods In Taiwan, Fisheries Agency provided compensation for maritime fatalities, and recorded all enrolled crews in Fishery Administration Management Information System. Using these two databases, incidence rate and odds ratio (OR) were calculated to depict an overall picture of maritime fatalities due to acute illness and associated causal factors.

Results From 2003 to 2015, there were 665 cases of maritime fatalities, whose mechanism were fatal accidents (562, 84.5\%) and acute illness $(103,15.5 \%)$. Overall incidence rate of maritime fatalities due to acute illness was 0.67 per 10000 manlabour year. The rates were $0.27,0.70$, and 2.58 per 10000 man-labour year, and odds ratios were 1.0, 2.60 and 9.65, for coastal $(<12$ Nautical miles, Nm), inshore $(12-200 \mathrm{Nm})$, and deep sea $(>200 \mathrm{Nm})$ fisheries.

Conclusion Our findings showed that one in seven maritime fatalities is due to acute illness in Taiwanese fishing vessels, and deep sea fisheries had the highest risk, followed by inshore and coastal fisheries, which may be explained by difference in accessibility to emergency medical care. This calls for intervention like regular health screening and occupational health service to prevent the occurrence of acute illness in advance, especially in deep sea fisheries.

\section{P.3.07 PROGNOSIS AND QUALITY OF LIFE IN WORKERS WITH OCCUPATIONAL UPPER LIMB INJURY}

Po-Ching Chu, Ching-Lin Hsieh, Yue Leon Guo. National Taiwan University and National Taiwan University Hospital, Taipei, Taiwan

\subsection{6/OEM-2019-EPI.270}

Objectives Because literatures about health impact of occupational upper limb injury in injured workers are rare, the study aimed to investigate effects of the injury on workers' prognosis and health-related quality of life.

Methods This was a cross-sectional study of 206 workers with occupational upper limb injury recruited from two teaching hospitals. Their sociodemographic factors, work-related factors, disease severity, return to work status, psychological symptoms, and quality of life were assessed by questionnaire interview at 2 years after injury.

Results The study found that the majority of injured workers were middle-aged (44.0 \pm 12.2 years) and men (58.3\%). There were $36.3 \%$ workers did not return to work at 2 year postinjury. The average EQ-5D was 0.81 (ranged from 0 to 1 ), and the psychological symptoms using 5-item Brief Symptom Rating Scale was 4.19 (ranged from 0 to 20). The multivariable linear regression showed that high educated injured workers had poorer quality of life $(\beta=-0.218, p=0.003)$ and more psychological symptoms $(\beta=6.35, p=0.001)$ than those with low education. In addition, workers with longer duration (months) of return to work had better quality of life $(\beta=0.0013, p=0.029)$ and lesser psychological symptoms $(\beta=-0.05, p=0.002)$, and the workers' compensation scheme 